\title{
CONSEQUÊNCIAS DA INTERAÇÃO ENTRE NUTRIÇÃO ENTERAL E FÁRMACOS ADMINISTRADOS POR SONDAS: UMA REVISÃO INTEGRATIVA*
}

\author{
Lolita Dopico da Silva ${ }^{1}$, Caroline de Deus Lisboa ${ }^{2}$
}

RESUMO: Trata-se de revisão integrativa que objetivou discutir os achados de publicações indexadas em bancos de dados, no período de 1999 a 2009, que abordam as consequências da interação entre fármacos e nutrição enteral administrados por sondas. A busca foi realizada no período de novembro a dezembro de 2009 nas bases MedLine, Lilacs e Scielo. A amostra final foi composta por sete artigos; houve predomínio de pesquisas apontando alterações na biodisponibilidade e absorção de medicamentos provocadas pela administração concomitante com a nutrição enteral. A maior parte dos artigos foi conclusiva em relação às interações, demonstradas pelos prejuízos aos pacientes que recebiam nutrição enteral e medicamentos concomitantemente.

PALAVRAS-CHAVE: Nutrição enteral; Interações alimento-droga; Enfermagem; Preparações farmacêuticas.

\section{CONSEQUENCES OF INTERACTION BETWEEN ENTERAL NUTRITION AND DRUGS ADMINISTERED BY CATHETER: AN INTEGRATIVE REVIEW*}

\begin{abstract}
An integrative review which aims to discuss the findings of publications indexed in data banks during the period 1999 to 2009 on the subject of interactions between drugs and enteral nutrition administered by catheters. The search was undertaken in November-December 2009 using the MedLine, Lilacs and Scielo data banks. The final sample consisted of seven articles, dominated by research pointing out alterations in bioavailability and absorption of medications caused by the concomitant administration of enteral nutrition. Most of the articles were conclusive in relation to the interactions, which were demonstrated by the harm caused to patients receiving both medication and enteral nutrition at the same time.

KEYWORDS: Enteral Nutrition; Drug-Food Interactions; Nursing; Pharmaceutical preparations.

\section{CONSECUENCIAS DE LA INTERACCIÓN ENTRE NUTRICIÓN ENTERAL Y FÁRMACOS ADMINISTRADOS POR SONDAS: UNA REVISIÓN INTEGRATIVA*}

RESUMEN: Esta es una revisión integrativa que tuvo el objetivo de discutir las informaciones de publicaciones indexadas en bancos de datos, en el periodo de 1999 a 2009, las cuales abordan las consecuencias de la interacción entre fármacos y nutrición enteral administrados por sondas. La búsqueda fue realizada en el periodo de noviembre a diciembre de 2009 en las bases MedLine, Lilacs y Scielo. La muestra final fue compuesta por siete artículos; hubo predominio de investigaciones que apuntaron alteraciones en la biodisponibilidad y absorción de medicinas, provocadas por la administración concomitante con la nutrición enteral. La mayor parte de los artículos fue conclusiva acerca de las interaciones, demostradas por los perjuicios a los pacientes que recebían nutrición enteral y medicinas concomitantemente. PALABRAS CLAVE: Nutrición enteral; Interacciones alimento-droga; Enfermería; Preparaciones farmacéuticas.

\footnotetext{
*Produto acadêmico do Programa de Pós-Graduação da Faculdade de Enfermagem da Universidade do Estado do Rio de Janeiro - PPGENF UERJ.

${ }^{1}$ Enfermeira. Pós-Doutora em Enfermagem. Professora do PPGENF UERJ.

${ }^{2}$ Enfermeira. Mestranda do PPGENF UERJ.
}

\author{
Autor correspondente: \\ Lolita Dopico da Silva \\ Universidade do Estado do Rio de Janeiro \\ R. Flordelice, 505 - 22753-800 - Rio de Janeiro-RJ-Brasil \\ E-mail: lolita.dopico@gmail.com
}

Recebido: $01 / 06 / 10$ Aprovado: $20 / 11 / 10$ 


\section{INTRODUÇÃO}

Na Unidade de Terapia Intensiva (UTI), frequente se encontra pacientes que recebem nutrição enteral por dispositivos como as sondas. Recebem, também, medicamentos por esta via, o que às vezes pode trazer complicações, como a obstrução da sonda e a interação entre o fármaco e os nutrientes da nutrição enteral, podendo levar a mudanças no efeito terapêutico esperado do medicamento ${ }^{(1)}$.

A Nutrição Enteral (NE) consiste em:

Alimento para fins especiais, com ingestão controlada de nutrientes, na forma isolada ou combinada, de composição definida ou estimada, especialmente formulada e elaborada para uso por sondas ou via oral, industrializado ou não, utilizado exclusiva ou parcialmente para substituir ou complementar a alimentação oral em pacientes desnutridos ou não, conforme suas necessidades nutricionais, em regime hospitalar, ambulatorial ou domiciliar, visando a síntese ou manutenção dos tecidos, órgãos ou sistemas ${ }^{(1: 3)}$.

A absorção dos nutrientes e de alguns fármacos ocorre por mecanismos semelhantes e, frequentemente, competitivos, apresentando como principal sítio de interação o trato gastrointestinal. Como exemplo, pode-se citar a interação entre a fenitoína e a nutrição enteral, em que ocorre a quelação entre o fármaco e cátions divalentes da fórmula enteral, bioinativação e incompatibilidade, tendo como consequência uma biodisponibilidade reduzida do medicamento ${ }^{(2)}$.

É comum, na prática diária junto a pacientes que recebem NE por sondas, que a Enfermagem prepare e administre medicamentos de apresentação sólida triturando os mesmos e, depois, diluindo em água para administrar pelas sondas, sem atentar se esses medicamentos poderiam ser triturados, se manterão suas propriedades, se a diluição está sendo feita com solução adequada, ou se os medicamentos estão sendo administrados em local correto para sua melhor absorção (estômago ou duodeno). Sabe-se que sondas com posicionamento gástrico favorecerão a absorção de fármacos dependentes de $\mathrm{pH}$ ácido, do mesmo modo que aquelas posicionadas em duodeno irão beneficiar a absorção de fármacos dependentes de $\mathrm{pH}$ alcalino.

A prática de triturar medicamentos, ainda frequente no contexto hospitalar, pode também ter como consequência a interação entre fármacos e a fórmula da NE, o que pode levar à diminuição da biodisponibilidade sérica e diminuição da concentração sérica máxima do fármaco e obstrução de sondas, como complicação dessa interação.

Cabe ao enfermeiro a supervisão e/ou administração de medicamentos e de NE. Portanto, este profissional deve conhecer os fármacos cuja velocidade de absorção e quantidade absorvida possam ser afetadas, ou não, pela presença de alimentos. Saber os aspectos envolvidos na interação fármaco-nutriente contribuirá para a avaliação e o controle dos resultados do cuidado de enfermagem aos pacientes que recebem medicamentos e NE, contribuindo para evitar interações que possam comprometer os resultados da terapia medicamentosa. Este profissional também pode fornecer subsídios para o desenvolvimento de protocolos a partir de grupos medicamentosos, o que contribuirá para a melhoria no cuidado aos pacientes.

Assim, este estudo teve por objetivo discutir os achados de publicações que abordam as consequências da interação entre fármacos e nutrição enteral administrados por sondas.

\section{METODOLOGIA}

Foi feita uma revisão integrativa em seis etapas: seleção da questão norteadora; definição das características das pesquisas primárias; seleção das pesquisas que compuseram a amostra; análise dos artigos; interpretação dos resultados; e o exame crítico dos achados, com a seguinte questão norteadora: "Quais os registros em publicações científicas das consequências da interação entre fármacos e nutrientes da NE quando ambos são administrados por sondas em pacientes adultos e hospitalizados?"

A identificação do objeto de estudo foi realizada incluindo todos os artigos sobre administração de medicamentos por sondas publicados no período de 10 de janeiro de 1999 a 20 de dezembro de 2009 e indexados no National Library of Medicine (Medline), Literatura Latino-Americana de Ciências da Saúde (Lilacs) e Scientific Electronic Library On Line (Scielo) visando atender a recomendação da literatura de que se busquem diferentes fontes para o levantamento de publicações. Os critérios de seleção foram: artigos em português, inglês e espanhol com os resumos disponíveis nas bases de dados supracitadas no período estabelecido; indexados pelos termos do mesh/desc: /interações alimento-droga/food-drug interactions/ interacciones alimento-droga; e nutrição enteral/ enteral nutrition/nutrición enteral; referirem-se à população adulta hospitalizada; abordar consequências da administração de medicamentos por sondas; tratar 
de pesquisa clínica ou de campo. Para coleta de dados, foi elaborado um instrumento baseado em protocolo de revisão bibliográfica, sendo substituídos os itens do original em inglês citation e interventions por "título" e "procedimentos metodológicos".

\section{RESULTADOS}

Foram identificados 71 artigos, dos quais foram selecionados sete. Seis artigos foram publicados em periódicos estrangeiros, sendo cinco norteamericanos e um espanhol; e um foi publicado em periódico nacional. Com relação à metodologia, todos têm abordagem quantitativa do tipo descritiva; nenhuma pesquisa multicêntrica. As principais técnicas de coleta de dados foram a análise de prontuário, formulários e observações não participantes.

Um estudo ${ }^{(3)}$ teve como objetivo relatar e discutir a interação envolvendo levodopa e proteínas na NE. Trata-se de um estudo de caso realizado com um paciente de 77 anos com Doença de Parkinson, admitido em uma UTI devido a hemorragia cerebral, sendo necessária a utilização de uma sonda orogástrica para administração de dietas e medicamentos. A diminuição do controle dos sintomas da Doença de Parkinson, apesar da utilização do medicamento, sugeriu fortemente a interferência da NE na absorção de levodopa, com a redução das concentrações plasmáticas máximas.

Estudo farmacocinético, prospectivo e randomizado $^{(4)}$, abordou a interação entre o antibiótico gatifloxacino e nutrientes da NE, e teve como objetivo comparar a administração do fármaco intravenoso e por sonda em relação à biodisponibilidade. Os resultados revelaram que a nutrição por sonda, concomitantemente à administração do antibiótico, não afetou a concentração plasmática do medicamento. A pesquisa revelou não haver consequências na biodisponibilidade do fármaco em pacientes que receberam gatifloxacino por sondas.

Uma outra publicação ${ }^{(5)}$ objetivou comparar a influência de interromper a infusão da nutrição enteral contínua por uma hora antes e uma hora depois da administração de warfarina, com a coadministração de warfarina com NE contínua a partir da avaliação da Razão Normalizada Internacional (INR). Trata-se de um estudo retrospectivo e de cruzamento de casos. Demonstrou que, quando a NE não foi interrompida para a administração do medicamento, o INR diminuiu em $73 \%$ comparado ao período em que foi interrompido. A consequência da interação foi a diminuição da biodisponibilidade do fármaco, causando o aumento do INR devido à redução da absorção da warfarina.

Outra pesquisa realizada ${ }^{(6)}$ teve por objetivo investigar se existe interação entre NE e warfarina; trata-se de dois casos clínicos e um estudo em laboratório. Os resultados evidenciaram que a warfarina se liga a proteínas da NE. Conclui-se que essa interação, clinicamente importante, ocorre quando a warfarina e a NE são administradas concomitantemente e a consequencia é a redução da biodisponibilidade do fármaco.

Outro estudo ${ }^{(7)}$ avaliou o uso de protocolos para manter a permeabilidade da sonda enteral em pacientes que requerem restrição de fluídos. A pesquisa considerou que a interação físico-química, provocada pela administração de medicamentos concomitante à NE, contribui para a obstrução da sonda. A intercorrência foi relacionada com a forma de apresentação dos medicamentos prescritos e a técnica de lavagem inadequada da sonda. Trata-se de uma pesquisa com desenho quase experimental, na qual 60 pacientes receberam NE por sonda, como parte de seu tratamento, e 5 a $7 \mathrm{ml}$ de água filtrada para irrigação das sondas. Para a implantação do protocolo foi feito um treinamento com a equipe de enfermagem, com o objetivo de, por meio da lavagem, evitar a obstrução da sonda, situação comumentemente evidenciada. $\mathrm{O}$ estudo concluiu que, após treinamento, houve melhora na compreensão de como proceder com pacientes em restrição hídrica.

O objetivo de outro estudo ${ }^{(8)}$ foi detectar as possíveis interações entre medicamentos e NE e descrever aquelas ações realizadas pelo farmacêutico; trata-se de um estudo prospectivo com pacientes que receberam NE. Foram realizadas 12 intervenções, sendo três para alteração de forma farmacêutica sólida para líquida, a fim de garantir a biodisponibilidade do medicamento; três por manipulação incorreta de formas farmacêuticas sólidas; três por interação fármaco/NE, para as quais foi proposta a interrupção por um hora antes e duas horas depois da administração do medicamento; uma intervenção por interação medicamento-medicamento; e três intervenções por interação físicoquímica por administrar medicamentos que podem precipitar se administrados concomitantemente com a NE e obstruir a sonda. Concluiu-se que as consequências da administração de medicamentos e NE podem levar à diminuição no organismo da concentração 
máxima esperada para determinado medicamento.

Outra pesquisa ${ }^{(9)}$ objetivou conhecer o padrão de preparo e administração de medicamentos a pacientes em uso de sondas para NE. Foram observados 60 auxiliares de enfermagem durante o preparo e administração de medicamentos. Os auxiliares realizaram trituração dos medicamentos na forma farmacêutica sólida em 90\% dos casos; 75\% lavaram a sonda após a administração do medicamento, apenas 5\% lavaram a sonda antes e depois da administração da medicação e $25 \%$ não lavaram a sonda. As consequências foram interações físico-químicas que podem levar à obstrução das sondas, à alteração na biodisponibilidade e diminuição da eficácia terapêutica do medicamento.

\section{DISCUSSÃO}

No estudo sobre levodopa ${ }^{(3)}$, o paciente tinha os sintomas da Doença de Parkinson sob controle e, ao receber doses do medicamento concomitante à NE, voltou a apresentar os sintomas da doença. Nesse caso, ocorreu uma interação farmacocinética entre proteínas da NE e o medicamento. Quando o teor de proteína da dieta enteral foi diminuído e separado da administração de levodopa, os sintomas da doença melhoraram. A levodopa é o agente mais eficaz no tratamento da Doença de Parkinson; quando administrada por via oral sofre rápida absorção pelo intestino delgado através de um sistema de transporte ativo de aminoácidos aromáticos. Sua administração concomitante com as refeições retarda a absorção e reduz suas concentrações plasmáticas máximas. A entrada do agente no sistema nervoso central, através da barreira hematoencefálica, também é um processo ativo mediado por um transportador de aminoácidos aromáticos, podendo ocorrer competição entre a proteína dietética e a levodopa nesse nível ${ }^{(10-11)}$.

Existem três estratégias disponíveis para diminuir o potencial de interação entre a NE e a levodopa. O primeiro método consiste em separar as fontes de proteína da administração da medicação; o segundo limita a quantidade total de ingestão diária de proteína e o terceiro método é aumentar a dose de levodopa. Para que a administração de proteínas e medicamentos ocorra separadamente, a recomendação inclui a administração de dietas em bolus durante horário noturno, para evitar que a administração de medicamentos ocorra no mesmo horário ${ }^{(3)}$. Porém, uma pesquisa ${ }^{(12)}$ evidenciou que $o$ aprazamento feito pelos enfermeiros ainda é pautado em rotinas institucionais e que existe pouca preocupação com as possíveis interações medicamentosas, indicando que ainda é insuficiente o seu conhecimento sobre farmacologia.

Os enfermeiros devem monitorar a resposta clínica do paciente para possíveis alterações sintomáticas da doença (efeitos liga/desliga, acinesias, tremores e rigidez) e evitar dietas hiperproteicas, impedindo que ocorra competição entre fármacos e proteínas da $\operatorname{dieta}^{(10,13)}$

A pesquisa ${ }^{(4)}$ realizada com o antibiótico gatifloxacino, amplamente utilizado para o tratamento de infecções do trato urinário, apontou que não houve mudança na biodisponibilidade do fármaco ao ser administrado concomitante à NE, de forma contínua ou pausada. Este achado pode ser atribuído ao fato deste antibiótico ser bem absorvido e distribuir-se amplamente pelos tecidos do corpo. A literatura evidencia que o alimento não compromete a absorção oral do fármaco, mas pode retardar o momento da concentração sérica máxima, alcançada entre 1 a 3 horas após uma dose oral de $400 \mathrm{mg}^{(10)}$.

Contrariamente, outro estudo ${ }^{(14)}$ discute a redução da absorção do fármaco pela interação com fórmulas de NE quando administrados concomitantemente, e recomenda que o antibiótico deva ser administrado somente uma hora antes e/ou duas horas depois da NE.

Dois artigos sobre anticoagulantes e $\mathrm{NE}^{(5-6)}$ demonstraram forte evidência de interação entre ambos com diminuição da biodisponibilidade da warfarina. Os anticoagulantes orais são utilizados para prevenir a progressão ou recidiva de trombose venosa profunda aguda e da embolia pulmonar, após um curso inicial de heparina. Além disso, são eficazes na prevenção da tromboembolia venosa em pacientes submetidos a cirurgia ortopédica ou ginecológica, bem como na prevenção da embolização sistêmica em pacientes com infarto agudo do miocárdio e próteses de valvas cardíacas $^{(10)}$.

Para a maioria das indicações, o alvo do Índice Internacional Normalizado (INR) é definido como alvo 2 a 3; porém, em geral, recomenda-se um INR alvo mais alto, por exemplo, 2.5 a 3.5, para pacientes com próteses mecânicas de valvas cardíacas ${ }^{(10)}$. O INR é definido como um índice do tempo de protrombina, obtido dividindo-se o tempo de protrombina com o tempo de tromboplastina controle, corrigido pelo Índice de Sensibilidade Internacional (ISI) ${ }^{(15)}$. As medidas diárias de INR devem ser realizadas pelo enfermeiro no início da terapia para evitar ocorrência 
de anticoagulação excessiva no paciente muito sensível $^{(16)}$. Esse intervalo será gradualmente aumentado até um intervalo semanal e, em seguida, mensal para pacientes submetidos a terapia prolongada ${ }^{(10)}$.

Em geral, a warfarina é administrada por via oral, porém, o fármaco também pode ser administrado por via intravenosa (IV), sem modificações da dose. Não se recomenda a injeção intramuscular(IM), devido ao risco de formação de hematoma. A biodisponibilidade da warfarina é quase completa quando administrada por via oral ${ }^{(10)}$. Entretanto, a presença de alimento no trato gastrointestinal (TGI) pode diminuir a taxa de absorção do fármaco e este fato pode explicar a diminuição da sua biodisponibilidade nos estudos encontrados. É uma importante evidência a necessidade de interrupção de dietas nos pacientes que utilizam a warfarina por sondas, para evitar que esta interação desencadeie uma diminuição do efeito terapêutico esperado $^{(17)}$. Em geral, a warfarina pode ser detectada no plasma dentro de uma hora após a sua administração oral, e obtém concentrações máximas dentro de 2 a 8 horas $^{(10)}$.

Alguns dos fatores mais comumente descritos e que provocam uma redução no efeito dos anticoagulantes orais incluem a redução da absorção do fármaco em decorrência da ligação à colestiramina no trato gastrointestinal; o aumento do volume de distribuição e meia-vida curta secundária à hipoproteinemia; o aumento da depuração metabólica do fármaco secundariamente à indução de enzimas hepáticas por barbitúricos, rifampicina, fenitoína ou ingestão crônica de álcool; e a ingestão de grandes quantidades de alimentos ou de suplementos ricos em vitamina $\mathrm{K}$. Por conseguinte, o tempo de protrombina diminui na maioria desses $\operatorname{casos}^{(10)}$.

Outras três pesquisas ${ }^{(7-9)}$ selecionadas abordaram as questões da interação físico-química entre NE e fármacos. Fármacos podem ter sua biodisponibilidade alterada por fatores como a presença de alimentos no estômago, incompatibilidade com o material da sonda $^{(18)}$ (como exemplo, as perdas de amiodarona para dispositivos confeccionados com PoliCloreto de Vinilo-PVC), e o preparo de medicamentos, como por exemplo, a trituração de comprimidos com revestimento entérico, na qual a alteração da forma farmacêutica prejudica sua eficácia terapêutica ${ }^{(19)}$.

Ao se preparar medicamentos para a administração por sondas, deve-se ter conhecimento de que formas farmacêuticas líquidas são as mais adequadas, pois elas são facilmente absorvidas e são as que menos causam obstrução de sondas ${ }^{(19)}$. Um estudo ${ }^{(9)}$ observou que os auxiliares de enfermagem preparam e administram medicamentos prescritos sem análise prévia da forma farmacêutica, dosagem da droga ou do potencial de interação. Quanto às formas líquidas, apesar de serem as formas mais apropriadas para preparo por sondas, deve-se saber que também apresentam limitações, como: problemas relacionados à viscosidade, à osmolaridade (muitas preparações líquidas são hiperosmolares) e aos excipientes existentes nas formulações, principalmente o sorbitol, o que aumenta o risco de intolerância do trato gastrointestinal ${ }^{(18)}$.

Já foi dito que a forma líquida é a mais adequada. Porém, quando não houver uma apresentação líquida disponível, as principais alternativas são utilizar outras vias de administração ou a troca por outro princípio ativo com atividade equivalente, que exista em formas líquidas ou que possa ser adaptado a essa via $^{(16)}$. Foi analisado um estudo ${ }^{(8)}$ no qual houve a troca de forma farmacêutica sólida por líquida; sugeriu-se alteração de digoxina em comprimido por digoxina suspensão, sendo reconhecida a relevância desta modificação por ser um fármaco com estreita margem terapêutica. O farmacêutico propôs o uso de forma líquida como alternativa ao uso de ácido valproico comprimido com revestimento entérico devido à impossibilidade de se triturar, já que a destruição do revestimento provocaria a inativação do fármaco e irritação da mucosa.

Ao se alterar a forma de um fármaco, a liberação segura do mesmo ao seu sítio de absorção pode não ocorrer. Fármacos que são revestidos entericamente para prevenir a sua destruição pelo ácido gástrico não devem ser triturados, pois o esmagamento destrói a capa entérica ${ }^{(19)}$. Há que se reconhecer que formas farmacêuticas sólidas, ao serem incorretamente trituradas, podem obstruir as sondas por fragmentos do medicamento que não formaram pó e, ainda, alguns fármacos podem ficar aderidos às sondas e formar um cimento no lúmen, causando também a oclusão.

Um estudo ${ }^{(20)}$ com objetivo de identificar os tipos de erros relacionados ao preparo e administração de medicamentos constatou que $17 \%$ dos erros estavam relacionados à diluição. Deve-se levar em consideração que uma diluição inadequada de medicamentos administrados por sondas, como já dito anteriormente, pode levar à sua obstrução, diarreias e ineficácia da terapêutica medicamentosa.

No material selecionado, são comentados alguns procedimentos que podem diminuir as interações 
entre fármacos e NE, tais como: nunca administrar medicamentos diretamente na fórmula de nutrição enteral; interromper a administração de nutrição enteral pelo menos 30 minutos antes e após administração de medicações; lavar a sonda com 15-30 $\mathrm{ml}$ de água antes e após administração de qualquer medicamento e entre os medicamentos; não associar dois tipos ou mais de medicamentos no processo de diluição; sempre que possível, administrar medicamentos na forma líquida, fato que evita a necessidade de modificação da forma de apresentação do medicamento como macerações de comprimidos.

\section{CONCLUSÃO}

Constataram-se publicações apontando como consequências da interação entre medicamentos e NE a redução na biodisponibilidade do fármaco (warfarina e levodopa) e a obstrução da sonda, o que não ocorreria se a administração de fármacos através de sondas fosse, preferencialmente, de medicamentos com apresentação líquida.

Cabe ao enfermeiro a supervisão do aprazamento, preparo e administração de fármacos e, também, do processo de terapia nutricional, enquanto responsável legal pela equipe de enfermagem. É necessário que este profissional tenha conhecimento acerca dos desdobramentos das interações entre fármacos e NE, de modo que ambas não sejam prejudicadas ou suprimidas, uma em detrimento da outra, a fim de se garantir a efetividade do tratamento a que o paciente tem direito. Logo, sugere-se que o enfermeiro realize revisão da prática de aprazamento dos fármacos e que realize educação em serviço referente à temática, em estudo junto aos profissionais sob sua responsabilidade.

Esta revisão aponta para a necessidade de se realizar estudos experimentais que utilizem misturas de fármacos com nutrição enteral, no intuito de aumentar a margem de segurança dos cuidados de enfermagem no âmbito medicamentoso, garantindo a eficácia da terapia enteral e farmacológica.

\section{REFERÊNCIAS}

1. Ministério da Saúde (BR). Regulamento Técnico para a Terapia de Nutrição Enteral. Resolução ANVS/DC/MS n.63/2000 de 06 de julho de 2000. Brasília; 2000.

2. Katzung BG. Farmacologia básica e clínica.10 ed. Rio de Janeiro: Rooca; 2007.

3. Cooper MK, Broock DG, Mc Daniel CM. Interactions between levodopa and enteral nutrition. Ann Pharmacother. 2008;42(3):439-42.

4. Kanji S, McKinnon PS, Barletta JF, Kruse JA, Devlin JW. Biovailability of gatifloxacin by gastric tube administration with and without concomitant feeding in critically ill patients. Crit Care Med. 2003;31(5):1347-52.

5. Dickerson RN, Garmon WM, Kuhl DA, Minard G, Brown RO. Vitamin K-independent warfarin resistence after concurrent administration of warfarin and continuos enteral nutrition. Pharmacotherapy. 2008;28(3):308-13.

6. Penrod LE, Allen JB, Cabacungan LR. Warfarin resistance and enteral feedings: 2 case reports and a supporting in vitro study. Arch Phys Med Rehabil. 2001;82(9):1270-3.

7. Matsuba CS, DeGutierrez MG, Whitaker IY. Development and evaluation of standardized protocol to prevent nasoenteral tube obstrution in cardiac patients requiring enteral nutrition with restricted fluid volumes. J Clin Nurs. 2007;16(10):1872-7.

8. Gago Sánchez AI, Almagro CGM, Aranzana C, Continente AC, Hernandez MAC. Atención farmacéutica en pacientes con nutrición enteral. Farm Hosp. 2006;30(1):44-8.

9. Heydrich J, Heineck I, Bueno D. Observação de preparação e administração de drogas por auxiliares de enfermagem em pacientes com tubo de alimentação enteral. Braz J Pharm Sci. [Internet] 2009;45(1):117120 [acesso em 20 out 2009]. Disponível: http://www. scielo.br/scielo.php?script=sci_arttext\&pid=S1984$82502009000100014 \& \operatorname{lng}=\mathrm{pt}$

10. Brunton L, Lazo S, Parker, K. As bases farmacológicas da terapêutica. 11ª ed. São Paulo: McGraw-Hill; 2007.

11. Lourenço R. Enteral feeding: drug nutrient interaction. Clin Nutr. 2001;20(2):187-93.

12. Fontenele RE, Araujo TL. Análise do planejamento dos horários de administração de medicamentos em unidade de terapia intensiva cardiológica. Rev Enferm UERJ. 2009;17(2):159-64.

13. Moura MRL, Reyes FG. Interação fármaco-nutriente: uma revisão. Rev Nutr. 2002;15(2):223-38. 
14. Engle KK, Hannawa TE. Techniques for administering oral medications to critical care pacients receiving continuous enteral nutrition. Am J Health - Syst Pharm. 1999;56:1441-4.

15. Hang HP, Dale MM. Farmacologia. $3^{\text {a }}$ ed. Rio de Janeiro: Guanabara Koogan; 2007.

16. Campanili TCGF, Ayoub AC. Warfarina: fatores que influenciam no índice de normatização internacional. Rev Eletr Enf. [Internet] 2008;10(4). [acesso em 25 jan 2009]. Disponível: http://www.fen.ufg.br/revista/v10/ n4/v10n4a19.htm.

17. Williams NT. Medication administration through enteral feeding tubes. Am J Health-Syst Pharm. 2008; 65(24):2347-57.

18. Clayton BD, Stock YN. Farmacologia na prática de enfermagem. 13 ${ }^{\mathrm{a}}$ ed. Rio de Janeiro: Elsevier; 2006.

19. Gámez L, Clópes EA, Cardona PD, Farré RR, Castro CI, Bonal FJ. Importancia de las características físicoquímicas de los fármacos para su administración por sonda nasoentérica o enterostomía. Farm Hosp.1998;22(3):137-43.

20. Praxedes MFS, Telles Filho PCP. Identificação de erros no preparo e administração de medicamentos pela equipe de enfermagem e das ações praticadas pela instituição hospitalar. Cogitare Enferm. 2008;13(4):5149. 